\title{
Toward Understanding Nucleosynthesis Patterns in Intermediate-Mass Stars: C, S, and Ar in PNe
}

\author{
R.B.C. Henry ${ }^{1}$, K.B. Kwitter ${ }^{2}$ and J. Buell ${ }^{1}$ \\ ${ }^{1}$ University of Oklahoma; ${ }^{2}$ Hopkins Observatory, Williams College
}

We report on our project to re-evaluate carbon and other abundances in PNe spanning the expected PN progenitor mass range. The ultimate goal of this work is three-fold: (i) to determine the abundance of carbon in this sample; (ii) to assess the abundances of sulfur and argon as metallicity indicators in intermediate-mass stars; and (iii) to incorporate our metallicity results into stellar evolution models to predict nucleosynthesis yields as a function of mass and metallicity.

For the carbon study, the UV data are taken from the recalibrated IUE Final Archive database. Optical data are taken from the literature; abundances are derived using a combination of a five-level-atom program and the CLOUDY modelling program (Ferland 1990, Ohio State University Report 90-02), as described in Henry, Kwitter \& Howard (1996, Ap.J., 458, 215). The general trend in PNe is confirmed: both $\mathrm{C} / \mathrm{O}$ and $\mathrm{N} / \mathrm{O}$ are enhanced relative to the sun. We also find that the halo PNe (BB-1, K648, H4-1) are systematically underabundant in oxygen, relative both to the sun and to the non-halo PNe.

To evaluate abundances of elements in PNe like sulfur and argon, which are expected to remain unaffected by nucleosynthesis by stars in this mass range, we have obtained new optical spectrophotometry for most of our sample objects. Ionization models predict more $\mathrm{S}^{++}$and $\mathrm{S}^{+3}$ than $\mathrm{S}^{+}$. In particular because most previous studies have used [S II] lines or the weak auroral $\lambda 6312$ line of [S III], we looked for [S III] $\lambda \lambda 9069,9532$. Our wavelength coverage also includes three dominant stages of argon: [Ar III] $\lambda 7135$, [Ar IV] $\lambda 4740$, and [ArV] $\lambda 7005$. We have preliminary results for five PNe, but with such a small data set no trend is evident. We plan to complete the necessary spectrophotometry in the near future, and the results for our entire sample will be published together.

Finally, we have calculated a series of models for K468 in the globular cluster M15. These are thermally-pulsing, asymptotic giant branch envelope models that include mass loss. The observed abundances in $\mathrm{K} 648$ can be matched with a single dredge-up episode of helium and carbon. Further details on the modelling procedure will be forthcoming (Buell et al., 1997, in preparation).

Acknowledgments We acknowledge support from NASA Grant NAG5-2389. K.B.K. also acknowledges support from the Research Corporation and from Williams College. R.B.C.H. thanks the Williams College Astronomy Department for its hospitality during several visits. 